\title{
Erratum to: Start-up and cessation Newtonian Poiseuille and Couette flows with dynamic wall slip
}

\author{
George Kaoullas · Georgios C. Georgiou
}

Published online: 30 December 2015

(C) Springer Science+Business Media Dordrecht 2015

\section{Erratum to: Meccanica (2015) 50:1747-1760 DOI 10.1007/s11012-015-0127-y}

The paper contains a number of typos as explained below. The correct versions of Eqs. (44)-(51) in Section 3 are as follows:

$$
\begin{aligned}
u_{x}^{*}\left(y^{*}, t^{*}\right) & =\frac{6}{1+3 B} \\
\sum_{n=1}^{\infty} & \frac{\sin \lambda_{n} \cos \left(\lambda_{n} y^{*}\right) e^{-\lambda_{n}^{2} t^{*}}}{\lambda_{n}^{2}\left(\frac{2 \lambda_{n} \Lambda^{*}}{B} \cos ^{2} \lambda_{n}+\lambda_{n}+\sin \lambda_{n} \cos \lambda_{n}\right)},
\end{aligned}
$$

The online version of the original article can be found under doi:10.1007/s11012-015-0127-y.

\section{G. Kaoullas $(\bowtie)$}

Oceanography Centre, University of Cyprus,

PO Box 20537, 1678 Nicosia, Cyprus

e-mail: g.kaoullas@gmail.com

\section{G. C. Georgiou}

Department of Mathematics and Statistics, University of Cyprus, PO Box 20537, 1678 Nicosia, Cyprus

e-mail: georgios@ucy.ac.cy

$$
\begin{aligned}
& u_{x}^{*}\left(y^{*}, t^{*}\right)= \frac{y^{*}+B}{1+2 B}-\sum_{n=1}^{\infty} C_{n}\left[\sin \left(\lambda_{n} y^{*}\right) .\right. \\
&\left.+\frac{B \lambda_{n}}{I} \cos \left(\lambda_{n} y^{*}\right)\right] e^{-\lambda_{n}^{2} t^{*}}, \\
& C_{n}=\frac{\left(B^{2} \lambda_{n}^{2}+I^{2}\right) \sin \lambda_{n}}{\lambda_{n}^{2} B\left(I^{2}+2 B+B^{2} \lambda_{n}^{2}+2 B \Lambda^{*} \lambda_{n}^{2}\right)}, \quad I=1-\lambda_{n}^{2} \Lambda^{*},
\end{aligned}
$$

$\tan \lambda_{n}=\frac{2 B I \lambda_{n}}{B^{2} \lambda_{n}^{2}-I^{2}}$

$$
\begin{aligned}
& u_{w 1}^{*}\left(t^{*}\right)=\frac{B}{1+2 B}-\sum_{n=1}^{\infty} C_{n} \frac{B \lambda_{n}}{I} e^{-\lambda_{n}^{2} t^{*}} \\
& u_{w 2}^{*}\left(t^{*}\right)=\frac{1+B}{1+2 B}-\sum_{n=1}^{\infty} C_{n}\left(\sin \lambda_{n}+\frac{B \lambda_{n}}{I} \cos \lambda_{n}\right) e^{-\lambda_{n}^{2} t^{*}}
\end{aligned}
$$

$$
Q^{*}\left(t^{*}\right)=\frac{1}{2}-\sum_{n=1}^{\infty} C_{n} \frac{1}{\lambda_{n}}\left(1-\cos \lambda_{n}+\frac{B \lambda_{n}}{I} \sin \lambda_{n}\right) e^{-\lambda_{n}^{2} t^{*}}
$$

$$
u_{x}^{*}\left(y^{*}, t^{*}\right)=\sum_{n=1}^{\infty} C_{n}\left[\sin \left(\lambda_{n} y^{*}\right)+\frac{B \lambda_{n}}{I} \cos \left(\lambda_{n} y^{*}\right)\right] e^{-\lambda_{n}^{2} t^{*}}
$$

Finally, Eqs. (72), (74) and (78) in Section 4 should read: 


$$
\begin{aligned}
L= & \frac{2 \Lambda v}{R^{2}} \kappa \lambda_{n}\left[Z_{1}^{2}\left(\lambda_{n}\right)+\kappa Z_{1}^{2}\left(\kappa \lambda_{n}\right)\right]+B \kappa \lambda_{n}\left[Z_{0}^{2}\left(\lambda_{n}\right)\right. \\
& \left.+Z_{1}^{2}\left(\lambda_{n}\right)-\kappa^{2}\left(Z_{0}^{2}\left(\kappa \lambda_{n}\right)+Z_{1}^{2}\left(\kappa \lambda_{n}\right)\right)\right] \\
& -2 B \kappa\left[Z_{0}\left(\lambda_{n}\right) Z_{1}\left(\lambda_{n}\right)-\kappa Z_{0}\left(\kappa \lambda_{n}\right) Z_{1}\left(\kappa \lambda_{n}\right)\right],
\end{aligned}
$$$$
\widetilde{D}_{n}=\frac{D_{n}}{\Omega \kappa R C},
$$

and

$$
u_{\theta}^{*}\left(r^{*}, t^{*}\right)=C \sum_{n=1}^{\infty} \widetilde{D}_{n} Z_{1}\left(\lambda_{n} r^{*}\right) e^{-\lambda_{n}^{2} t^{*}} .
$$

We would like to thank Professor Chiu-On $\mathrm{Ng}$ (University of Hong Kong) for pointing out some of the typos. 